\title{
Behaviour of Solutions of a Third Order Differential Equation
}

\author{
M. GREGUŠ and D. ŠišoLÁKOVÁ
}

Properties of proper and regular non-oscillatory solutions of the equation $x^{\prime \prime \prime}+f(t) g\left(x, x^{\prime}, x^{\prime \prime}\right)=$ 0 , and some sufficient conditions for regular solutions to be oscillatory or non-oscillatory are derived.

Key words: Proper solutions, regular solutions, oscillatory and non-oscillatory solutions, thind order differential equations

AMS subject classification: $34 \mathrm{C} 15$

1. We will deal with the differential equation

$$
x^{\prime \prime \prime}+f(t) g\left(x, x^{\prime}, x^{\prime \prime}\right)=0,
$$

where

(i) $f$ is continuous on $(a,+\infty), a>-\infty$, and $f(t)>0$ for all $t \in(a,+\infty)$

(ii) $g$ is continuous on $R^{3}$ and $g\left(y_{0}, y_{1}, y_{2}\right)>0$ for all $\left(y_{0}, y_{1}, y_{2}\right) \in R^{3}$ with $y_{0} \neq 0$.

Equation (1) is a generalization of equations examined in $[1,2]$ and a special case of equations examined in $[4,5]$.

2. The aim of this paper is to investigate so-called proper and regular solutions of equation (1).

Definition 1: A function $x \in C^{3}\left(T_{x},+\infty\right)\left(T_{x} \geq a\right)$ being a solution of equation (1) is said to be a

a) proper sulution if $\sup \{|x(t)|: t \geq T\}>0$ for every $T>T_{x}$;

b) regular solution if it is proper and if for some $T \geq T_{x}$ it does not have a triple null point in $(T,+\infty)$, i.e. there is no $t_{0} \geq T$ such that $x\left(t_{0}\right)=x^{\prime}\left(t_{0}\right)=x^{\prime \prime}\left(t_{0}\right)=0$;

c) oscillatory solution if it is proper or regular and if there exists a sequence $t_{i} \uparrow+\infty$ such that $x\left(t_{i}\right)=0$ for all $i$;

d) non-oscillatory solution if it is not oscillatory, i.e. if there exists a $T \geq T_{x}$ such that $x(t)>0$ for all $t>T$ or $x(t)<0$. for all $t>T$.

M. Gregư⿱ Univ. J.A. Komenský, Dep. Math., Mlynská dolina, 842 is Bratislava. Slovakia D. Sižoláková: Univ. J.A. Komenský, Dep. Math., Mlynská dolina, 84215 Bratislava, Slovakia 
Theorem 1: Let $x$ be a proper solution of equation (1) on $\left\langle T_{x},+\infty\right)$ and let $\rho>T_{x}$ be a triple null point of $x$. Then there exists an $\epsilon>0$ such that on $(\rho, \rho+\epsilon)$ either $x=0$ or $x \neq 0$ and $x$ has infinite many null points with $\rho$ as limiting point.

Proof: It is sufficient to prove that there does not exist an $\epsilon>0$ such that $x(t) \neq 0$ for all $t \in(\rho, \rho+\epsilon)$. Suppose $x(t)>0$ for all $t \in(\rho, \rho+\epsilon)$. Then $x^{\prime \prime \prime}(t)<0$ for all $t \in(\rho, \rho+\epsilon)$ and $x^{\prime \prime}$ is decreasing. But $x^{\prime \prime}(\rho)=0$ and therefore $x^{\prime \prime}(t)<0$ for all $t \in(\rho, \rho+\epsilon)$. In the same way we get $x^{\prime}(t)<0$ and $x(t)<0$ for all $t \in(\rho, \rho+\epsilon)$ which leads to a contradiction. In the case $x(t)<0$ for all $t \in(\rho, \rho+\epsilon)$ the proof is similar

Theorem 2: Let $f$ and $g$ be functions satisfying conditions (i) and (ii), respectively. Moreover, let there exists for any $\left(y_{0}, y_{1}, y_{2}\right) \in R^{3}$ with $y_{0} \neq 0$

$$
\text { (iii) } g_{1}\left(y_{1}, y_{2}\right):=\lim _{y_{0} \rightarrow 0} \frac{g\left(y_{0}, y_{1}, y_{2}\right)}{y_{0}}
$$

and let the function $g_{1}$ be continuous on $R^{2}$. Then the trivial solution $x=0$ of equation (1) fulfilling the initial conditions $x\left(t_{0}\right)=x^{\prime}\left(t_{0}\right)=x^{\prime \prime}\left(t_{0}\right)=0\left(t_{0}>a\right)$ is unique on $\left(t_{0},+\infty\right)$

Proof: Let $x$ be a solution of equation (1) with $t_{0}$ as triple null point and $x(t) \neq 0$ in $\left(t_{0}, t_{0}+\epsilon\right)$ for some $\epsilon>0$. Then $x$ fulfils also the equation

$$
u^{\prime \prime \prime}(t)+f(t) G(t) u=0
$$

on $\left(t_{0}, t_{0}+\epsilon\right)$, where

$$
G(t)= \begin{cases}g\left(x(t), x^{\prime}(t), x^{\prime \prime}(t)\right) / x(t) & \text { for } x(t) \neq 0 \\ g_{1}\left(x^{\prime}(t), x^{\prime \prime}(t)\right) & \text { for } x(t)=0\end{cases}
$$

Equation (2) is linear and therefore it has only one solution with $t_{0}$ as triple null point, namely $u=0$. But this is in contradiction with the supposition of $x$

Corollary 1: Let $x$ be a proper solution of equation (1) on $\left(T_{x},+\infty\right)$. Then the following statements are true.

a) The non-trivial solution $x$ has at most one double or triple null point.

b) If $x(t)=c(c \in R)$ on some subinterval $I$, then $c=0$.

c) If $t_{1}, t_{2}$ are triple null points of $x$ with $t_{1}<t_{2}$, then $x=0$ on the interval $\left\langle t_{1}, t_{2}\right\rangle$.

d) The solution $x$ cannot be zero on any different disjoint subintervals $I_{1}$ and $I_{2}$.

Proof: a) Let $t_{0}>T_{x}$ be a double null point of $x$ and $t_{1}>t_{0}$ its next double null point. Denote $F(x)=x x^{\prime \prime}-x^{\prime 2} / 2$. Then multiplying equation (1) by $x$ and integrating from $t_{0}$ to $t$ we obtain

$$
F(x(t))=\int_{t_{0}}^{t} f(\tau) g\left(x(\tau), x^{\prime}(\tau), x^{\prime \prime}(\tau)\right) x(\tau) d \tau=F\left(x\left(t_{0}\right)\right)
$$

Because $t_{0}, t_{1}$ are double null points the function $F(x(\cdot))$ is non-increasing for $t \geq t_{0}$ and $F\left(x\left(t_{0}\right)\right)=F\left(x\left(t_{1}\right)\right)=0$ which contradicts that $x$ is a non-trivial solution of equation (1). The case of a solution $x$ with triple null point can be proved by analogous arguments.

b) Let $t_{0} \in I$ and $c \neq 0$. Then from (4) we get $F(x(t))=-\int_{t_{0}}^{t} f(\tau) g(c, 0,0) c d \tau<0$ for $t>t_{0}$ and $F(x(\cdot))$ is non-increasing. But $F(x(t))=F(c)=0$ which and is possible for $c=0$ only. 
c) - d) The proof of these suppositions follows immediately from the monotonicity of the function $F(x(\cdot))$ d

3. In this section we will be interested in non-oscillatory solutions of equation (1).

Lemma 1. Let $x$ be a solution of equation (1) on $\left(T_{x},+\infty\right)$ without null points and with $F(x(t))>0\left(t \geq t_{1}\right)$ for the function $F(x(\cdot))$ defined by (4). Then there exists a point $T \geq T_{x}$ such that, for $t>T$, the functions $x, x^{\prime}, x^{\prime \prime}$ are monotone, have no null point, and either

$$
\operatorname{sgn} x(t)=\operatorname{sgn} x^{\prime \prime}(t) \neq \operatorname{sgn} x^{\prime}(t) \quad \text { or } \quad \operatorname{sgn} x(t)=\operatorname{sgn} x^{\prime \prime}(t)=\operatorname{sgn} x^{\prime}(t)
$$

Proof: From (4) it follows that $x^{\prime \prime}(t) \neq 0$ and $\operatorname{sgn} x(t)=\operatorname{sgn} x^{\prime \prime}(t)$ for $t \geq T_{x}$. From the properties of $x$ and $x^{\prime \prime}$ it follows that $x^{\prime}$ is monotone for $t \geq T_{x}$ and that it can have at most one null point. Therefore there exists a point $T \geq T_{z}$ such that either $\operatorname{sgn} x^{\prime}(t)=\operatorname{sgn} x(t)$ or $\operatorname{sgn} x^{\prime}(t) \neq \operatorname{sgn} x(t)$ for $t \geq T$. It remains to prove that $x^{\prime \prime}$ is monotone for $t \geq T$. From $x(t) \neq 0$ it follows that $x^{\prime \prime \prime}(t)=-f(t) g\left(x(t), x^{\prime}(t), x^{\prime \prime}(t)\right) \neq 0$ for $t \geq T_{x}$, that is $x^{\prime \prime \prime}(t)$ does not change the sign and therefore $x^{\prime \prime}$ is monotone

Theorem 3: Let $x$ be a solution of equation (1) with $x(t) \neq 0$ and $F(x(t))>0$ for all $t \in\left\langle T_{x},+\infty\right)$. Let further $\int_{t_{0}}^{+\infty} f(t) d t=+\infty$ for $t_{0}>a$ and

$$
\liminf _{t \rightarrow \infty} \varphi(t) g\left(\varphi(t), \varphi^{\prime}(t), \varphi^{\prime \prime}(t)\right)>0 .
$$

for any function $\varphi \neq 0$ with $\lim _{t \rightarrow+\infty} \varphi(t)= \pm \infty$ and which is monotone with $\varphi^{\prime}$ and $\varphi^{\prime \prime}$ on $(a,+\infty)$. Then there exists $a T \geq T_{x}$ such that, for $t>T$,

$$
x(t) x^{\prime}(t) x^{\prime \prime}(t) \neq 0 \quad \text { and } \quad \operatorname{sgn} x(t)=\operatorname{sgn} x^{\prime \prime}(t) \neq \operatorname{sgn} x^{\prime}(t)
$$

and $x(t), x^{\prime}(t), x^{\prime \prime}(t) \rightarrow 0$ as $t \rightarrow+\infty$.

Proof: From Lemma 1 it follows that $x, x^{\prime}, x^{\prime \prime}$ are monotone on $\left(T_{x},+\infty\right)$ and therefore having proper or improper limits at infinity. Let e.g. $\lim _{t \rightarrow+\infty} x(t) \neq 0$. Then clearly by (5), or directly by (ii), if $\lim _{t \rightarrow+\infty} x(t)$ is finite, then $\liminf \operatorname{in}_{t \rightarrow+\infty} x(t) g\left(x(t), x^{\prime}(t), x^{\prime \prime}(t)\right) \geq$ $c>0$. From (4) for $x$ there follows the existence of a $T \geq T_{x}$ such that, for $t>T, F(x(t)) \uparrow$ and

$$
F(x(t))-F(x(T))=-\int_{T}^{t} f(s) x(s) g\left(x(s), x^{\prime}(s), x^{\prime \prime}(s)\right) d s<0 .
$$

On the other hand we have

$$
F(x(t))-F(x(T))=-\int_{T}^{t} f(s) x(s) g\left(x(s), x^{\prime}(s), x^{\prime \prime}(s)\right) d s \leq-c \int_{T}^{s} f(s) d s .
$$

From here for $t \rightarrow+\infty$ we obtain a contradiction to $F(x(t))>0$ for $t>T_{x}$ and therefore $x(t) \rightarrow 0$ for $t \rightarrow+\infty$. From the properties of $x$ there follows $\operatorname{sgn} x(t) \neq \operatorname{sgn} x^{\prime}(t)$ for $t \geq$ $T^{\prime} \geq T$, where $T^{\prime}$ is sufficiently large. From $F(x(t))>0$ there follows $\operatorname{sgn} x(t)=\operatorname{sgn} x^{\prime \prime}(t)$ and from the properties of $x$ there follows $x^{\prime}(t), x^{\prime \prime}(t) \rightarrow 0$ as $t \rightarrow+\infty$

Example 1: Let us have the differential equation

$$
x^{\prime \prime \prime}+x \frac{\arctan \sqrt{1+x^{2}+x^{\prime 2}+x^{\prime \prime 2}}}{\arctan \sqrt{1+3 e^{-2 t}}}=0
$$


for $t \in(0,+\infty)$. This equation fulfils the suppositions of Theorem 3 . Its every solution $x$ defined on $\left\langle T_{x},+\infty\right)$ for some $T_{x}>0$, with $x(t) \neq 0$ and $F(x(t))>0$ for all $t \in\left\langle T_{x},+\infty\right)$ has the property $x(t) x^{\prime}(t) x^{\prime \prime}(t) \neq 0, \operatorname{sgn} x(t)=\operatorname{sgn} x^{\prime \prime}(t) \neq \operatorname{sgn} x^{\prime}(t)$ for $t \in\langle T,+\infty)(T \geq$ $T_{x}$ ) and $x(t), x^{\prime}(t), x^{\prime \prime}(t) \rightarrow 0$ as $t \rightarrow+\infty$. The function $x=e^{-t}$ is clearly a solution of this equation.

Lemma 2 (see [6]): Let $h \in C(a,+\infty)$ and $\int_{t_{0}}^{+\infty} t^{2} h(t) d t<+\infty, t_{0}>a$. Then the differential equation

$$
y^{\prime \prime \prime}+h y=0
$$

is non-oscillatory on $(a,+\infty)$, i.e. every solution of equation (6) has a finite set of null points on $(a,+\infty)$.

Theorem 4: Let $f$ and $g$ be functions satisfying conditions (i) - (iii) and let $x$ be a solution of equation (1) on $\left(T_{x},+\infty\right)$ such that

$$
\left|g\left(x(t), x^{\prime}(t), x^{\prime \prime}(t)\right)\right| \leq|x(t)| \varphi(t) \quad\left(t>t_{0}\right)
$$

for some positive function $\varphi \in C\left(t_{0},+\infty\right)\left(t_{0}>T_{x}\right)$ satisfying the condition

$$
\int_{t_{0}}^{+\infty} t^{2} f(t) \varphi(t) d t<+\infty
$$

Then $x$ is non-oscillatory on $\left\langle T_{0},+\infty\right)$.

Proof: Suppose that $x$ is oscillatory. Then $x$ is also a solution of equation (2) and from our suppositions it follows that $\int_{t_{0}}^{+\infty} t^{2} f(t) G(t) d t \leq \int_{t_{0}}^{+\infty} t^{2} f(t) \varphi(t) d t<+\infty$. Therefore from Lemma 2 it follows that equation (2) is non-oscillatory. This is a contradiction with the supposition that $x$ is oscillatory

Example 2: Let us have the differential equation

$$
x^{\prime \prime \prime}+\frac{1}{t^{4}} \frac{\arctan x}{1+x^{2}+x^{\prime 2}+x^{\prime \prime 2}}=0
$$

for $t \in(0,+\infty)$. This equation fulfils the suppositions of Theorem 4 for $\varphi(t)=M>$ $\pi / 2(t \in(0,+\infty))$. Therefore any of its solution on $\left\langle t_{0},+\infty\right)\left(t_{0}>0\right)$ is non-oscillatory.

Corollary 2: Let $f$ and $g$ be functions satisfying (i) - (iii) and such that (8) holds for $\varphi=1$. Then equation (1) has no oscillatory solution $x$ bounded with $x^{\prime}, x^{\prime \prime}$ on $\left(t_{0},+\infty\right), t_{0}>a$.

Proof: Let $x$ be a solution of equation (1) on $\left\langle T_{x},+\infty\right)$ and let $x, x^{\prime}, x^{\prime \prime}$ be bounded on this interval. Then from the continuity of $g$ and from supposition (iii) there follows the existence of a constant $M>0$ such that $0 \leq G(t) \leq M$ for all $t \in\left(T_{x},+\infty\right)$ where $G$ is given by (3). Then the assertion follows from Theorem 4 for $\varphi=M$

Remark: Theorem 4 and Corollary 2 generalize the results of Theorem 3 and of Corollary 2 of [1].

4. We deduce in this section sufficient conditions for the oscillatory and non-oscillatory nature of solutions of equation (1) by means of the so-called generalized Kneser criterion 
(for second order linear differential equations) generalized in [3] for the linear equation (6).

Lemma 3: Let $0 \leq h \in C(0,+\infty)$ and $t_{0}>0$.

a) If $h \geq 0, h(t) \leq \frac{2}{3 \sqrt{3}} \frac{1}{t^{3}}$ for $t \in\left\langle t_{0},+\infty\right)$ and $h \neq 0$ on any subinterval of $\left(t_{0},+\infty\right)$, then equation (6) is not oscillatory on $\left(t_{0},+\infty\right)$.

b) If there is a $\delta>0$ such that $h(t) \geq \frac{1}{3}(1+\delta) \sqrt{\frac{1}{3}(4+\delta)} \frac{1}{t^{3}}$ for $t \in\left(t_{0},+\infty\right)$, then equation (6) is oscillatory on $\left(t_{0},+\infty\right)$, i.e. every solution of equation (6) with a null point in $\left(t_{0},+\infty\right)$ oscillates on this interval.

Proof: The proof is given in [3] as proof of Corollary 2.7

Theorem 5: Let $f$ and $g$ be functions satisfying conditions (i) $-($ iii) on $(0,+\infty)$ and let $\delta, t_{0}>0$.

a) If for all $\left(y_{0}, y_{1}, y_{2}\right) \in R^{3}$ and $t \in\left(t_{0},+\infty\right)$

$$
f(t)\left|g\left(y_{0}, y_{1}, y_{2}\right)\right| \leq \frac{2}{3 \sqrt{3}} \frac{1}{t^{3}}\left|y_{0}\right|
$$

then equation (1) does not have an oscillatory solution defined on $\left(t_{0},+\infty\right)$.

b) If for all $\left(y_{0}, y_{1}, y_{2}\right) \in R^{3}$ and $t \in\left(t_{0},+\infty\right)$

$$
f(t)\left|g\left(y_{0}, y_{1}, y_{2}\right)\right| \geq \frac{1}{3}(1+\delta) \sqrt{\frac{1}{3}(4+\delta)} \frac{1}{t^{3}}\left|y_{0}\right|,
$$

then any solution $x$ of equation (1) defined on $\left(t_{0},+\infty\right)$ and having a null point is oscillatory on the right of this null point.

Proof: a) Under our conditions $x$ is a solution of equation (2) on $\left(t_{0},+\infty\right)$ and by (9) satisfies the inequality $f(t) G(t) \leq \frac{2}{3 \sqrt{3}} \frac{1}{t^{3}}$ for all $t \in\left(t_{0},+\infty\right)$, where $G$ is given by (3). Now from Lemma 3 it follows that equation (2) is non-oscillatory, $x$ is a non-oscillatory solution of equation (2) and of equation (1), too. The assertion b) can be proved with the same arguments as in the foregoing assertion

The proof of the next lemma is given in [3: Theorem 3.6].

Lemma 4: Let $h \in C(a,+\infty)$ and $d>0$ be such that $h(t) \geq d$ for all $t \in(a,+\infty)$. Then every solution of equation (6) is oscillatory in $(a,+\infty)$ except solutions $y$ (unique up to linear dependence) satisfying the conditions

$$
\int_{a}^{+\infty} y^{2}(t) d t<+\infty \quad(a<\alpha<+\infty) \quad \text { and } \quad y, y^{\prime} \rightarrow 0^{\circ} \text { as } t \rightarrow+\infty \text {. }
$$

Theorem 6: Let $x$ be a solution of equation (1) on $\left(T_{x},+\infty\right)$, let the functions $f$ and $g$ satisfy the conditions $(i)-(i i i)$ and

$$
f(t)\left|g\left(y_{0}, y_{1}, y_{2}\right)\right| \geq M\left|y_{0}\right| \quad(M>0)
$$

for arbitrary $\left(y_{0}, y_{1}, y_{2}\right) \in R^{3}$ and $t \in(a,+\infty)$.

a) If $x \neq 0$, then $\int_{T_{x}}^{+\infty} x^{2}(t) d t<+\infty$ and $x, x^{\prime} \rightarrow 0$ as $t \rightarrow+\infty$.

b) If $x$ has a null point, then $x$ is oscillatory. 
Proof: a) The function $x$ fulfils equation (2) and by (11) we bave the inequality $f(t)\left|g\left(x(t), x^{\prime}(t), x^{\prime \prime}(t)\right)\right| \geq M|x(t)|$ which yields $f(t) G(t) \geq M$. By Lemma 4 equation (2) is oscillatory on $\left(T_{x},+\infty\right)$ and $x$ is its solution without null points. Again by Lemma 4 it must have the properties stated. Assertion b) follows immediately by Theorem 5

Lemma 5: Let $h \in C(a,+\infty)$ and $h(t)>0$ for all $t \in(a,+\infty)$.

a) If equation (6) has an oscillatory solution, then every solution but one (up to linear dependence) is oscillatory and the non-oscillatory solution $y$ has the properties $y(t) \neq$ $0, \operatorname{sgn} y(t)=\operatorname{sgn} y^{\prime \prime}(t) \neq \operatorname{sgn} y^{\prime}(t)$ for all $t \in(a,+\infty), y, y^{\prime}, y^{\prime \prime}$ are monotone on $(a,+\infty)$ and $y^{\prime}(t), y^{\prime \prime}(t) \rightarrow 0$ as $t \rightarrow+\infty$.

b) If $y$ is a monotone solution of $(6)$ and $y(t) \neq 0$, sgn $y(t)=\operatorname{sgn} y^{\prime \prime}(t) \neq \operatorname{sgn} y^{\prime}(t)$ for all $t \in(a,+\infty)$, then $y(t) \rightarrow 0$ as $t \rightarrow+\infty$ if and only if $\int_{t_{0}}^{+\infty} t^{2} h(t) d t=+\infty$.

Proof: The proof of statement a) is given in [3: Theorem 2.13] and that of statement b) is given in [7]

Theorem 7: Let $f$ and $g$ be functions satisfying the properties (i) - (iii) and (10). Then every solution $x$ of equation (1) with the property $x(t) \neq 0$ in $\left(T_{x},+\infty\right)$ tends to zero as $t \rightarrow+\infty$.

Proof: The function $x$ fulfils equation (2). By Theorem 5 this equation is oscillatory (i.e. every solution with a null point in $\left(T_{x},+\infty\right)$ is oscillatory). By Lemma 5/a) equation (2) has only one solution without null points in $\left(T_{x},+\infty\right)$. Therefore this solution must be $x$ and it has the properties as in Lemma 5/a). It follows from (10) that $\int_{T_{x}}^{+\infty} t^{2} f(t) G(t) d t=$ $+\infty$ and therefore by Lemma $5 / \mathrm{b}) x(t) \rightarrow 0$ as $t \rightarrow+\infty$ D

The equation in Example 1 fulfils the suppositions of Theorem 7 on $\left\langle t_{0},+\infty\right)$ for sufficiently large $t_{0}>0$ and $x=e^{-t}$ is its solution with $x(t) \neq 0$ for $t>t_{0}$.

\section{References}

(1) Cecchi, M. and M. Marini: On the oscillatory behaviour of a thind onder nonlinear differential equation. Nonlin. Anal. 15 (1990), 141 - 153.

[2] Gregus, M.: On a nonlinear binomial equation of the third order. Z. Anal. Anw. 11 (1991), 117 124.

[3] Gregui, M.: Thind Onder Linear Differential Equations. Dortrecht et al: D. Reidel Publ. Comp. 1987.

[4] Śvec, M.: Monotone solutions of some differential equations. Colloq. Math. 43 (1967), 7 - 21.

[5] Švec, M.: Behaviour of nonoscillatory solutions of some nonlinear differential equations. Acta Math. Univ. Comen. 39 (1980), $115-130$

[6] Villari, G.: Sul carattere oscillatorio delle soluzioni delle equazioni differenziali lineari omogenee del terzo ondine. Boll. Un. Mat. Ital. 13 (1958), 73 - 78.

[7] Villari, G.: Contributi allo studio asintotico dell' equazione $z^{\prime \prime \prime}+p(t) x=0$. Ann. Mat. Pura Appl. 51 (1960), $301-328$. 